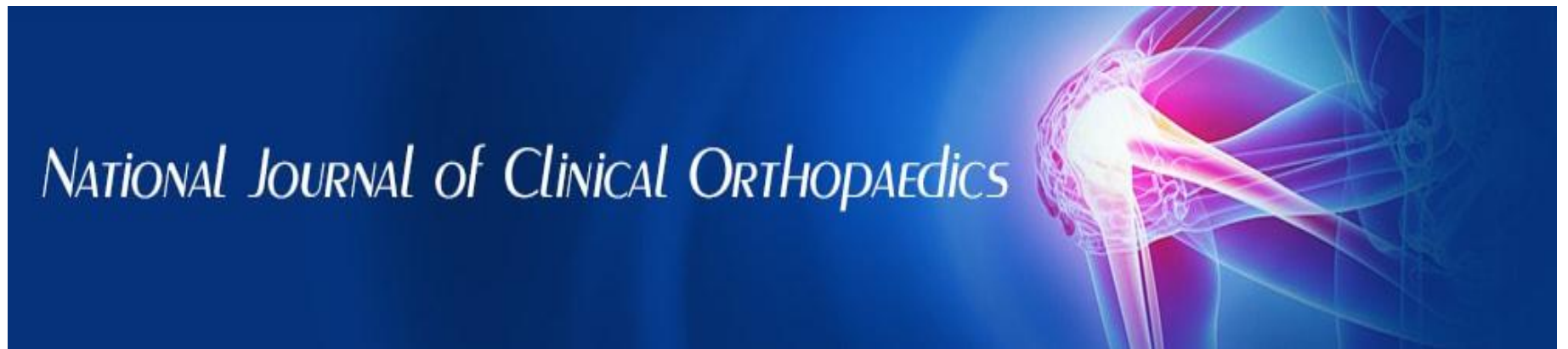

ISSN (P): 2521-3466

ISSN (E): 2521-3474

(C) Clinical Orthopaedics

www.orthoresearchjournal.com

2020; 4(1): 09-14

Received: 07-11-2019

Accepted: 10-12-2019

Dr. Nitin Manral

Senior Resident, Department of Orthopaedics KGMC Lucknow,

Uttar Pradesh, India

\section{Dr. Dharmender}

Senior Resident, Department of Orthopaedics Batra Hospital and Medical Research Centre New

Delhi, India

\section{Dr. Aditya Mongia}

Senior Resident, Department of Orthopaedics Batra Hospital and Medical Research Centre New Delhi, India

\section{Dr. Mohit}

Junior Resident, Department of Orthopaedics Batra Hospital and Medical Research centre New Delhi, India
Corresponding Author: Dr. Nitin Manral

Senior Resident, Department of Orthopaedics KGMC Lucknow, Uttar Pradesh, India

\section{Effect of acetabular cup orientation and femoral stem placement on the functional outcome in uncemented total hip arthroplasty}

\author{
Dr. Nitin Manral, Dr. Dharmender, Dr. Aditya Mongia and Dr. Mohit
}

DOI: https://doi.org/10.33545/orthor.2020.v4.i1a.193

\section{Abstract}

Background: A total joint arthroplasty refers to non-biologic replacement of articulating surfaces of a joint. The positioning of the acetabular component is a crucial step of the surgical technique. The position of the acetabular component in the medial-lateral direction influences offset leading to changes in the moment-lever arm of the abductor musculature. The anteversion of the acetabular cup in the sagittal plane and the inclination angle (also known as abduction angle) in the coronal plane are both important determinants of acetabular position.

Aim: The present study was conducted to see the effect of acetabular cup orientation and femoral stem placement on the functional outcome in cementless hip replacement arthroplasty exclusively.

Methodology: The present open-ended retrospective study was conducted in the Department of Orthopaedic Surgery, King George Medical University, Lucknow, UP on patients operated by uncemented total hip arthroplasty from 1st January 2012 to 30th April 2015. The following protocol was followed for every patient: -

- Demographic data and clinical examination of enrolled patients was noted.

- Functional outcome assessment by calculating the Harris Hip Score

- Radiological Evaluation by a standard x-ray of pelvis with both hip-AP (including whole implant) Statistical tools: Final data was analyzed by SPSS v. 16. Normality of the data described by mean and SD. For comparing variables with normal distribution, paired t-test, independent t-test, and ANOVAs test were used. Qualitative variables were expressed with frequency and percent. A $p$ value of $<0.05$ was considered statistically significant.

Results: In our study we found that the mean Harris hip score increased from 42.5 points (range 18-69) preoperatively to 94.3 points(range, $82-100$ ) at mean follow up of 2 years ( $p$ value-<0.001). Our study showed excellent functional outcome in 31 hips $(81.5 \%)$ while 7 hips $(18.5 \%)$ had good functional outcome.

Conclusion: In our study we did not find any effect of femoral stem placement on the functional outcome. Whether the stem is implanted in varus, valgus or neutral alignment, no significant difference was seen in their mean Harris Hip Scores.

Keywords: Total Hip Arthroplasty, Harris hip score, acetabular cup, femoral stem

\section{Introduction}

Total Hip Arthroplasty (THA) is a joint reconstruction procedure. The term "arthroplasty" is derived from $\operatorname{Arthr}(\mathrm{o})$ - refers to the procedure as it is "related to a joint" while -plasty from Greek origin means "to form". Since the introduction of low- friction arthroplasty, other modern improvements in metallurgy, tribology, surgical techniques and overall success of THA has led this procedure to be named the "Operation of the 20th Century" in 2007 [1].

Total hip replacement (THR) development began with cementless THR. McKee and WatsonFarrar documented an early model of an artificial hip joint between 1956 and1960. Charnley systematically promoted THR based on the concept of low friction arthroplasty by ${ }^{[2]}$.

- fixation with bone cement;

- Adoption of a 22-mm femoral head;

- Adoption of ultra-high molecular weight polyethylene (UHMWPE); and

- Preparation of a manual of the surgical procedure. 
A THA consists of a femoral component, acetabular component and a bearing surface. The technique to affix components without the use of cement relies initially on an interference fit, while osseous integration of the implant into host bone provides eventual fixation after the initial phase. Patients report significant improvements in their physical health in the postoperative period, especially within the first six months ${ }^{[3]}$.

Pain scores have been shown to improve the most within a short period of time. Physical function has been demonstrated to improve significantly after THA when compared to preoperative functioning. Long-term studies have shown that these improvements are not short- lived ${ }^{[4,5]}$.

The positioning of the acetabular component is a crucial step of the surgical technique. The position of the acetabular component in the medial-lateral direction influences offset leading to changes in the moment-lever arm of the abductor musculature. The anteversion of the acetabular cup in the sagittal plane and the inclination angle (also known as abduction angle) in the coronal plane are both important determinants of acetabular position. Improper acetabular orientation has been linked to increased dislocation rates ${ }^{[6,10]}$, polyethylene wear ${ }^{[11]}$, edge loading [12], liner fracture ${ }^{[13]}$, and component impingement affecting range of motion.

In a long-term retrospective review of the cemented Charnley total hip at 16-25 years postoperative, Devitt and colleagues ${ }^{[14]}$ determined a $75 \%$ survival rate of the implant at 20 years postoperative. For the stems placed in varus, the authors cite a $35.7 \%$ revision rate.

Despite the poor results of cemented varus stems, few studies to date have addressed the question of the effects of varus alignment on cementless stems. Thus, in our study we have tried to see the effect acetabular cup orientation and femoral stem placement has on the functional outcome in cementless hip replacement arthroplasty exclusively.

\section{Materials and Method}

The retrospective study was conducted in the Department of Orthopaedic Surgery, King George Medical University, Lucknow, UP. It was an open-ended retrospective study. Patients admitted through orthopedics OPD in King George's Medical University, Lucknow and operated by uncemented total hip arthroplasty from 1 st January 2012 to 30 th April 2015 in the Department of Orthopaedic surgery KGMU Lucknow were included in the study as per following inclusion and exclusion criteria.

\section{Inclusion Criteria}

- Patient undergone primary uncemented total hip arthroplasty from 1st January 2012 to 30 th april 2015 in KGMU.

- Minimum follow-up of one year

- Patient giving informed consent.

\section{Exclusion criteria}

- Patient undergone revision THR

- Patients with debilitating medical comorbidities interfering with functional outcome assessment.

- Patient not willing to participate in the study.

- Patients undergone hybrid total hip arthroplasty.

The patients who met the inclusion criteria were recalled in orthopaedics OPD once for follow up and following protocol was followed for every patient: -

1. Demographic data of enrolled patients was noted.

2. Clinical examination of the patient was done.

a. General Examination

b. Systemic Examination

c. Local Examination

3. Functional outcome assessment by calculating the Harris Hip Score

4. Radiological Evaluation by a standard x-ray of pelvis with both hip-AP (including whole implant)

\section{Statistical Tools}

Final data was analyzed by SPSS v. 16. Normality of the data described by mean and SD. For comparing variables with normal distribution, paired t-test, independent t-test, and ANOVAs test were used. Qualitative variables were expressed with frequency and percent. A p value of $<0.05$ was considered statistically significant.

\section{Results}

In present study average age of the patients was $30.74 \pm 7.8$ years and maximum number of patients $59.26 \%(n=16)$ belonged to the age group 21-30 years. Minimum age of patient was 20 years and maximum age was 48 years. Male predominance was seen in our study i.e. 25 of our patients were male $(92.6 \%)$ and 2 were female $(7.4 \%)$ In our study we found that the mean Harris hip score increased from 42.5 points (range 18-69) preoperatively to 94.3 points post operatively (range, 82-100) at mean follow up of 2 years.

\section{Acetabular cup inclination}

In our study, 5 acetabular cups were close cups, 5 were open cups and 28 cups were neutral. The Harris Hip score was calculated for these hips and was compared.

Table 1: Acetabular cup inclination and functional outcome of different acetabular cup inclination

\begin{tabular}{|c|c|c|c|c|c|}
\hline Inclination & No. of hips & Percentage & Mean Harris Hip Score & $\begin{array}{c}\text { Follow Up Time <=24 } \\
\text { Months }\end{array}$ & $\begin{array}{c}\text { Follow Up Time }>24 \\
\text { Months }\end{array}$ \\
\hline$<35^{\circ}$ (Closed cup) & 05 & 13.16 & $92.6 \pm 6.77$ (EXCELLENT) & $\mathrm{N}=1$ & $\mathrm{~N}=4$ \\
\hline $35^{\circ}-55^{\circ}$ (Neutral cup) & 28 & 73.68 & $95.14 \pm 3.18$ (EXCELLENT) & $\mathrm{N}=21$ & $\mathrm{~N}=7$ \\
\hline$>55^{\circ}$ (Open cup) & 05 & 13.16 & $91.6 \pm 4.27$ (EXCELLENT) & $\mathrm{N}=3$ & $\mathrm{~N}=2$ \\
\hline
\end{tabular}

$\mathrm{N}$ is number of hips

The mean Harris hip score for these 3 acetabular cup inclinations was calculated. The functional outcome as determined by the mean Harris hip score was similar i.e. Excellent in all the 3 acetabular cup inclinations. 


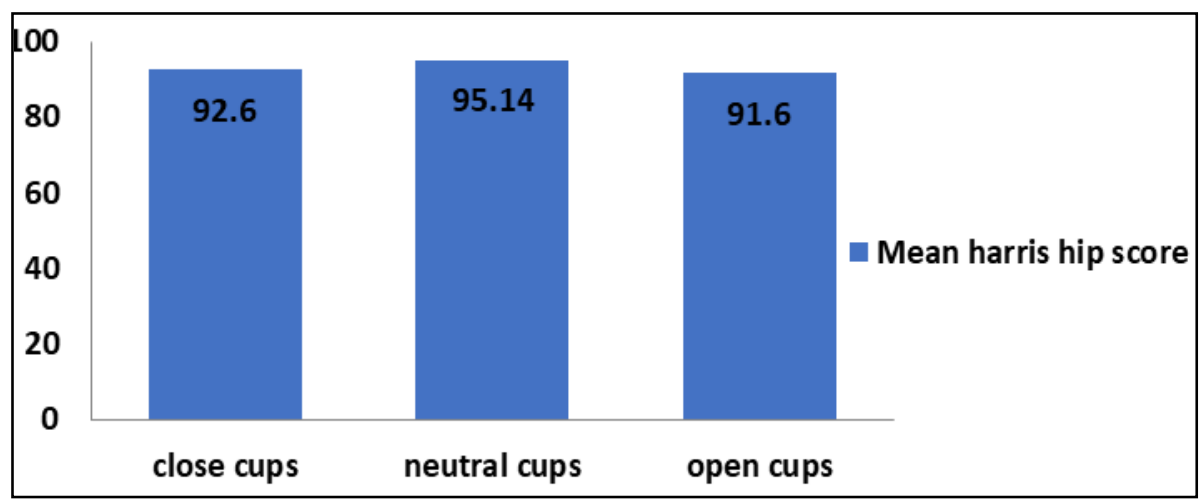

Fig 1: Mean Harris Hip score for different acetabular cup inclination

The mean Harris hip score \pm standard deviation was calculated for each cup orientation i.e. close, neutral and open in both the groups i.e. patients with follow up interval less than or equal to 24 months and patients with follow up interval greater than 24 months and the mean Harris hip scores for these three-cup inclinations were compared and p-value calculated. (Table 4)

Table 2: Mean Harris Hip Score for different inclination of acetabular cup

\begin{tabular}{|c|c|c|c|c|}
\hline \multirow[b]{2}{*}{$\begin{array}{l}\text { Follow Up } \\
\text { Time }\end{array}$} & \multicolumn{3}{|c|}{ Inclination } & \multirow[b]{2}{*}{$\begin{array}{c}\text { P- } \\
\text { Value }\end{array}$} \\
\hline & \begin{tabular}{|c|} 
Close Cup \\
$($ mean HHS \pm SD $)$
\end{tabular} & $\begin{array}{c}\text { Neutral Cup } \\
(\text { mean HHS } \pm \text { SD) }\end{array}$ & $\begin{array}{c}\text { Open Cup } \\
(\text { mean HHS } \pm \text { SD })\end{array}$ & \\
\hline$<=24$ Months & $82 \pm 0(\mathrm{~N}=1)$ & $94.0 \pm 4.53(\mathrm{~N}=21)$ & $88.7 \pm 2.08(\mathrm{~N}=03)$ & 0.061 \\
\hline$>24$ Months & $95.3 \pm 3.77(\mathrm{~N}=4)$ & $94.8 \pm 3.12(\mathrm{~N}=7)$ & $96.0 \pm 0(\mathrm{~N}=02)$ & $t$ \\
\hline
\end{tabular}

$\mathrm{N}=$ number of hip

There is no significant difference ( $p$ value-0.061) between the functional outcome of patients with neutral cup (mean Harris hip score- 94 \pm 4.53 ) than patients with open cup (mean Harris hip score-88.7 \pm 2.08 ) and in patients with close cup (mean Harris hip score- 82 ) in patients with less than 24 months follow up.
Similarly there is no significant difference ( $p$ value-0.896) in functional outcome in patients with greater than 24 months follow up. All three inclination groups have excellent outcome with close cups having mean Harris hip score of 95.3 \pm 3.77 , neutral cups having $94.8 \pm 3.12$ and open cups having 96.0 (Table 2, Figure 2)

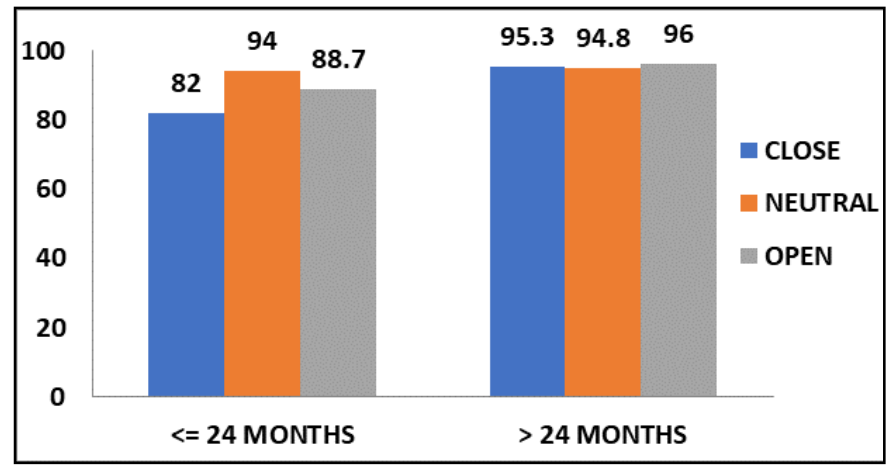

Fig 2: Mean Harris hip score of different acetabular cup inclination

Table 3: Distribution of Acetabular cup anteversion and Mean Harris Hip score for different anteversion of acetabular cup:

\begin{tabular}{|c|c|c|c|c|c|}
\hline Anteversion & No. of hips & Percentage & Mean Harris Hip score & Follow Up Time < =24 Months & Follow Up Time $>24$ Months \\
\hline$<5^{\circ}$ (low) & 04 & 10.5 & $96 \pm 1.41$ (EXCELLENT) & $\mathrm{N}=4$ (Mean \pm SD) $96 \pm 1.41$ & $\mathrm{~N}=0$ (Mean \pm SD) NA \\
\hline $5^{\circ}-25^{\circ}$ (normal) & 30 & 78.9 & $93.8 \pm 4.3$ (EXCELLENT) & $\mathrm{N}=19$ (Mean \pm SD) $93.16 \pm 4.81$ & $\mathrm{~N}=11$ (Mean \pm SD) $94.9 \pm 3.14$ \\
\hline$>25^{\circ}$ (high) & 04 & 10.5 & $96.75 \pm 2.22$ (EXCELLENT) & $\mathrm{N}=2$ (Mean \pm SD) $98 \pm 2.83$ & $\mathrm{~N}=2(\mathrm{Mean} \pm \mathrm{SD}) 95.5 \pm 0.71$ \\
\hline & & & & $\mathrm{P}$ value 0.23 & $\mathrm{P}$ value 0.79 \\
\hline
\end{tabular}

$\mathrm{N}=$ number of hip

In our study 4 acetabular cups had angle of anteversion less than $5^{\circ}, 30$ acetabular cups had anteversion between $5^{\circ}-25^{\circ}$ and 4 acetabular cups had anteversion greater than $25^{\circ}$. The mean Harris hip score for these 3 acetabular cup anteversion was calculated. The functional outcome as determined by the mean Harris hip score was similar i.e. Excellent in all the 3 groups of acetabular cup anteversion. (Table 3, Figure 3)

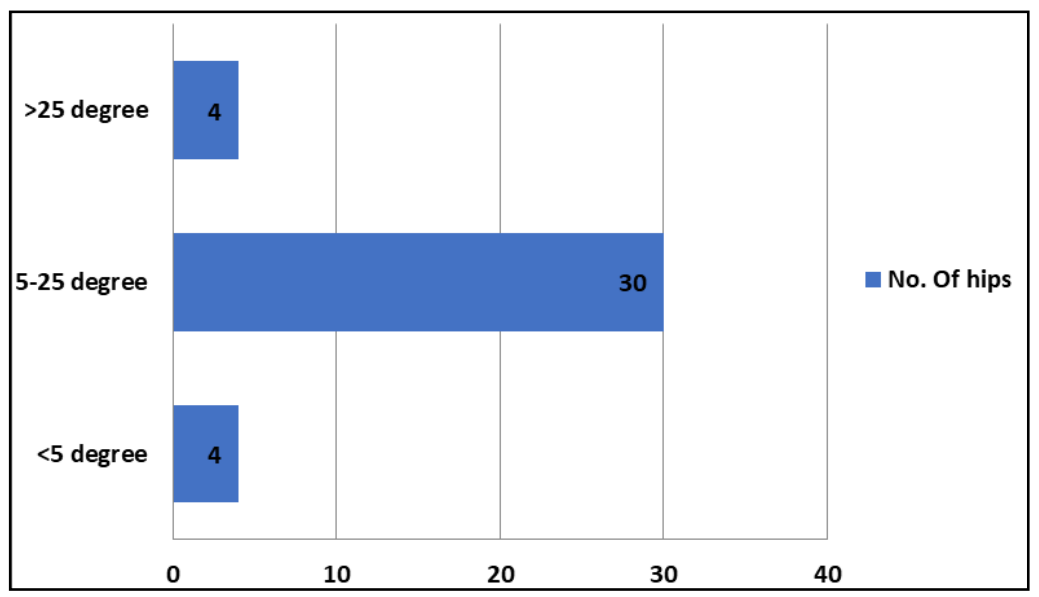

Fig 3a: Distribution of patients with different acetabular cup anteversion 
No significant difference(p value-0.23) between the functional outcome with low anteversion of acetabular cup (mean Harris hip score-96 \pm 1.41$)$ as compared to normal anteversion of acetabular cup (mean Harris hip score-93.16 \pm 4.81 ) or high anteversion of the acetabular cup (mean Harris hip score$98 \pm 2.83$ ) in patients with follow up less than or equal to 24 months. Similarly in patients with follow up greater than 24 months no significant difference( $p$ value-0.79) is seen in functional outcomes in acetabular cups with normal anteversion (mean Harris hip score-94.9 \pm 3.14 ) compared to acetabular cups with high anteversion (mean Harris hip score-95.5 \pm 0.71 ).(Table 3, Figure 3b)

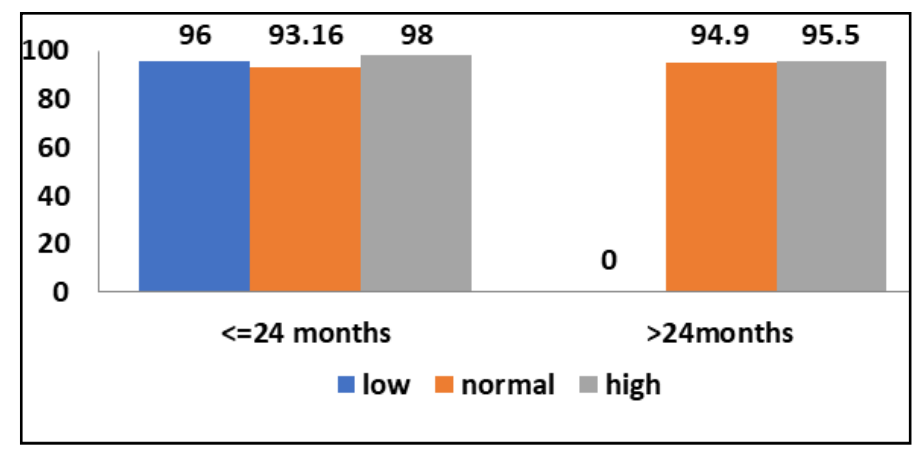

Figure 3b: Mean Harris Hip Score for different anteversion of acetabular cup

Femoral Stem placement

In our study 2 femoral stems were in varus, 31 femoral stems were in neutral and 5 stems were in valgus. (Table4, Figure 4)

Table 4: Femoral Stem placement and their Mean Harris Hipscore

\begin{tabular}{|c|c|c|c|c|c|}
\hline Stem Placement & No. of stems & Percentage & Mean Harris Hipscore & Follow Up Time <=24 Months & Follow Up Time $>24$ Months \\
\hline Varus & 02 & 5.3 & $96 \pm 0.0$ (Excellent) & $\mathrm{N}=0$ (Mean \pm SD) NA & $\mathrm{N}=2(\mathrm{Mean} \pm \mathrm{SD}) 96 \pm 0$ \\
\hline Neutral & 31 & 81.6 & $94.29 \pm 4.28$ (Excellent) & $\mathrm{N}=23$ (Mean \pm SD) $93.9 \pm 4.7$ & $\mathrm{~N}=8(\mathrm{Mean} \pm \mathrm{SD}) 95.5 \pm 2.56$ \\
\hline Valgus & 05 & 13.1 & $94 \pm 3.39$ (Excellent) & $\mathrm{N}=2$ (Mean \pm SD) $95.5 \pm 0.7$ & $\mathrm{~N}=3$ (Mean $\pm \mathrm{SD}) 93 \pm 4.36$ \\
\hline & & & & $\mathrm{P}$ value 0.635 & $\mathrm{P}$ value 0.257 \\
\hline
\end{tabular}

No significant difference ( $p$ value-0.635) between the functional outcome with neutral placement of femoral stem (mean Harris

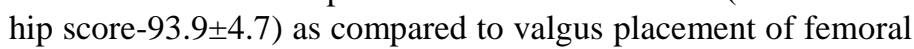

stem (mean Harris hip score-95.5 \pm 0.7 ) in patients with follow up less than or equal to 24 months.

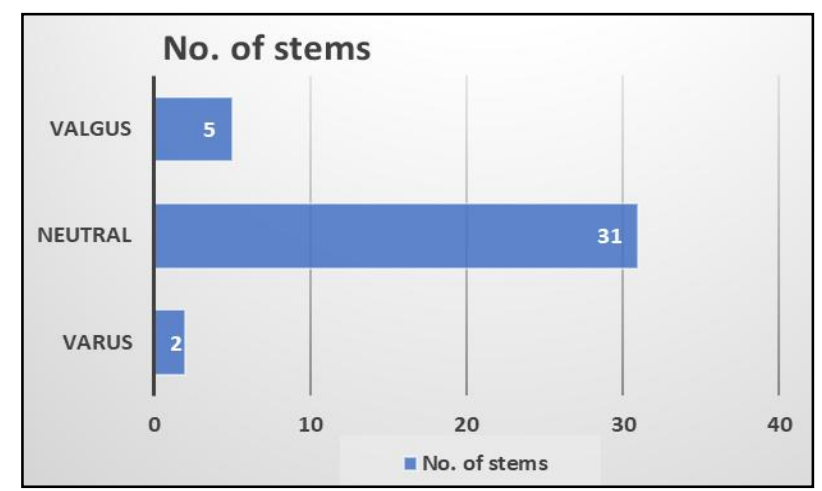

Fig 4a: Distribution of hips on basis of femoral stem placement

Similarly in patients with follow up greater than 24 months no significant difference ( $p$ value-0.257) is seen in functional outcomes in femoral stems with varus placement (mean Harris hip score-96 \pm 0 ) compared to neutral stem placement (mean Harris hip score-95.5 \pm 2.56 ) and valgus stem placement (mean

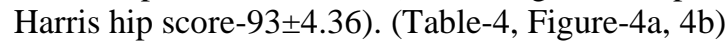

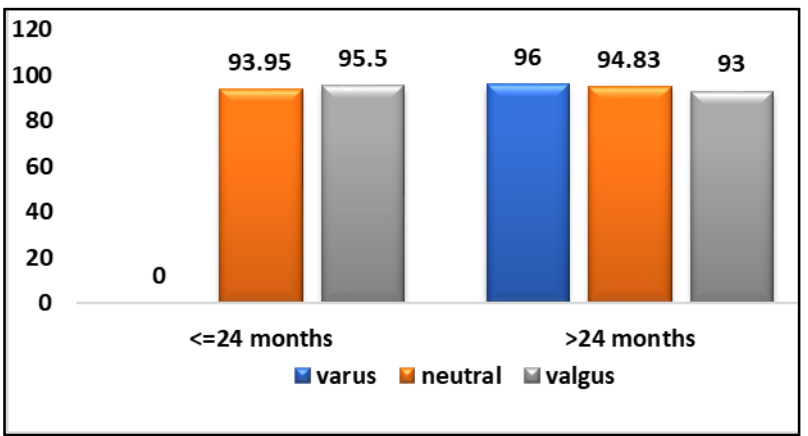

Fig 4b: Mean Harris Hip Score for different femoral stem placement 
Patients with different acetabular anteversions or inclination have similar functional outcome. Similarly patients with different femoral stem placement also have similar functional outcome. Thus we can say that femoral stem placement has no effect on the functional outcome at a mean follow up of 24 months.

\section{Heterotopic ossification}

Heterotopic ossification usually first becomes visible on radiographs three to four weeks after surgery and matures by three to six months. The incidence ranges from $5 \%$ to $90 \%$ in various literatures. In our study 7 hips (18\%) showed heterotopic ossification. They were classified according to classification given by Brooker. (Figure-5)

Grade I = 4 Hips

Grade II = 2 Hips

Grade III = 1 Hip

Grade IV $=$ Nil

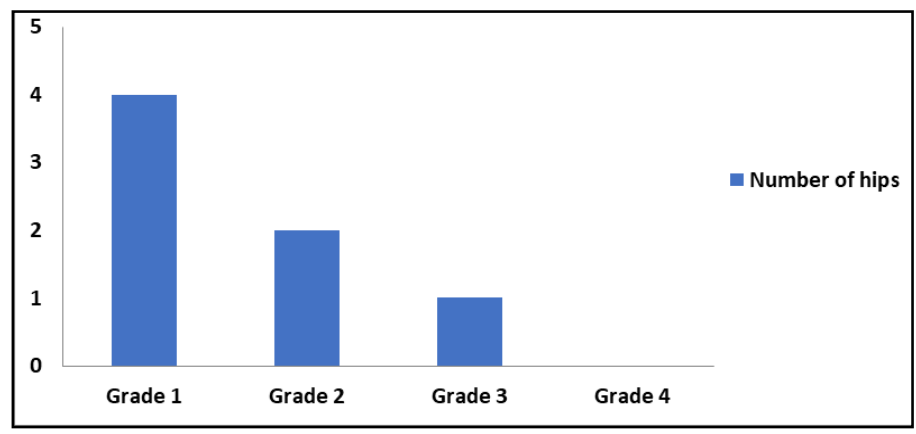

Fig 5: Chart showing patients with different grades of heterotopic ossification

Stress shielding- Osteopenia of proximal femur especially greater trochanteric region due to stress shielding appeared in $5 \mathrm{~s}$ tems $(13 \%)$. These femurs had a stem that was well integrated in the host tissue. No case with calcar resorption or pedestal formation was seen.

\section{Subsidence-Subsidence of femoral component was not seen}

Loosening-Loosening was seen in one case in the acetabular component with no evidence of acetabular migration and with no clinical significance.

\section{Discussion}

In our study, the majority of the patients were in the age group of 21-30 years. There were $16(59.26 \%)$ patients in this age group. The mean age of the patients in our study was $30.74 \pm 7.8$ years with youngest patient being 20 years old and maximum age of patient was 48years. Our results are also comparable to study of Kawalkar et al ${ }^{[15]}$. who reported the average age to be 27 years (range 20-39 years). In our study we found a male predominance in terms of sex distribution. There were 25 male patients $(92.6 \%)$ and 2 female patients $(7.4 \%)$. Our results are in agreement with the study of Kawalkar AC et al ${ }^{[15]}$, in which 17 $(68 \%)$ were males and $8(32 \%)$ were females thus showing a male preponderance. In our study 13 patients had been operated on left hip followed by 11 patients who were operated bilaterally and 3 patients operated on right hip. Majority of patients included in our study had avascular necrosis (14 patients, 52\%) of femoral head followed by ankylosing spondylitis (10 patients, $37 \%$ ) followed by post traumatic osteoarthritis (3 patients, 11\%). Out of the 14 patients with avascular necrosis, 4 patients were operated bilaterally, and remaining 10 patients with avascular necrosis of femoral head were operated unilaterally, 2 were operated on right hip and 8 were operated on left hip. All 3 patients with post traumatic arthritis were operated on left hip. Among the 10 patients with ankylosing spondylitis 7 patients were operated bilaterally and out of remaining 3,1 was operated on right hip and 2 were operated on left hip. Thus out of the total 38 hips operated, 24 were left sided, 14 were right sided among them 11 patients had bilateral THR.

In our study we found that the mean Harris hip score increased from 42.5 points (range 18-69) preoperatively to 94.3 points(range, 82-100) at mean follow up of 2 years (p value$<0.001)$. Our study showed excellent functional outcome in 31 hips $(81.5 \%)$ while 7 hips $(18.5 \%)$ had good functional outcome. The mean angle of inclination in our study was $44.4 \pm 9$ degrees. The range of inclination was 23-68 degrees. In our study we did not find any significant difference in the functional outcome as calculated by Harris Hip Score for different angles of inclination. In or study 5 acetabular cups (13.2\%) were close cups, 5 acetabular cups(13.2\%) were open cups and 28 acetabular cups(73.6\%) were neutral with mean Harris hip score of $92.6 \pm 6.77$ for close cups, $95.14 \pm 3.18$ for neutral cups and $91.6 \pm 4.27$ for open cups. All of these groups had excellent functional outcome scores which were in accordance with Justin de Beer et al ${ }^{[5]}$.

In our study, mean angle of anteversion was $12.4 \pm 6.7$ degrees. The range of anteversion was 4-27degrees. We did not find any significant difference in the functional outcome as calculated by Harris hip score in different angles of anteversion. In our study 4 acetabular cups $(10.5 \%)$ had angle of anteversion less than $5^{\circ}, 30$ acetabular cups $(78.9 \%)$ had anteversion between $5^{\circ}-25^{\circ}$ and 4 acetabular cups $(10.5 \%)$ had anteversion greater than $25^{\circ}$ with Harris Hip Score of $96 \pm 1.41$ for low angle of anteversion, $93.8 \pm 4.3$ for normal angle of anteversion, and $96.75 \pm 2.22$ for high angle of anteversion. All of these groups had excellent functional outcome scores. Our study is consistent with findings of Goyal et al [3], They studied 1,241 hips with 502 males (40.5\%) and 739 females (59.5). The mean absolute position of the population included $21.7 \pm 11.9$ degrees of anteversion and $44.4 \pm 6.9$ degrees of inclination angle. The range of inclination angle was 20.0 to 69.5 degrees. In our study we did not find any effect of femoral stem placement on the functional outcome. Whether the stem is implanted in varus, valgus or neutral alignment, no significant difference was seen in their mean Harris Hip Scores.

\section{Conclusion}

Based on our study we conclude that -

- Uncemented total hip arthroplasty is indicated in younger patients with good bone stock with excellent functional outcome and less complications in short term follow up. The mean age of patient operated was $30.74 \pm 7.8$ years

- Majority of Indian patients undergoing uncemented THR suffer from secondary osteoarthritis caused by avascular necrosis of femoral head and inflammatory arthropathies.

- Uncemented THR has shown excellent functional outcome in patients at a mean follow up of 2 years.

- The acetabular component orientation i.e. acetabular cup inclination and acetabular cup orientation has no effect on the functional outcome of the patients at a mean follow up of 2 years though a larger sample size and longer duration of follow up is needed to validate the findings and look for long term effect and complications.

- The femoral component alignment also does not affect the 
patient perceived functional outcome at a mean follow up of two years though a larger sample size and longer duration of follow up is needed.

- Loosening of femoral stems was not encountered at 'mean follow up of 2 years, however loosening of acetabular component was seen in one patient. Most of the uncemented implants showed stable osseous fixation of the stem and the cup and loosening was not frequent.

- Heterotopic ossification and stress shielding was seen in around $15 \%$ of patients.

\section{There are however certain limitations of our study}

1. Sample size was too small for comparison and a greater sample size would allow for better predictions and results.

2. The follow up period was too short, a longer follow up would allow for better assessment of the outcome and in finding out long term complications.

\section{References}

1. Learmonth ID, Young C, Rorabeck C. The operation of the century: total hip replacement. The Lancet, 2007. 370(9597):1508-19

2. Harumoto Yamada et al. Cementless total hip replacement: past, present, and future J Orthop Sci. 2009; 14(2):228-241

3. Goyal Prateek. "Effects of Acetabular Positioning in Total Hip Arthroplasty" Electronic Thesis and Dissertation Repository. Paper 3273, 2015.

4. Angadji A, Royle M, Collins SN, Shelton JC. Influence of cup orientation on the wear performance of metal-on-metal hip replacements Proc Inst MechEng H. 2009; 223(4):44957.

5. Justin de Beer, Scott McKenzie, Matthias Hubmann, Danielle Petruccelli, Mitchell Winemaker. Influence of cementless femoral stems inserted in varus on functional outcome in primary total hip arthroplasty Can J Surg. 2006; 49(6):407-411.

6. Lewinnek GE et al. Dislocations after total hip-replacement arthroplasties. J Bone Joint Surg Am. 1978; 60(2):217-20.

7. Ali Khan M, Brakenbury P, Reynolds I. Dislocation following total hip replacement. J Bone Joint Surg Br. 1981; 63-B(2):214-218

8. Robinson $\mathrm{M}$ et al. Effect of restoration of combined offset on stability of large head THA. Hip Int. 2012; 22(3):248-53

9. Jolles BM, Zangger P, Leyvraz PF. Factors predisposing to dislocation after primary total hip arthroplasty: a multivariate analysis. J Arthroplasty. 2002; 17(3):282-8.

10. Daines BK, Dennis DA. The importance of acetabular component position in total hip arthroplasty. OrthopClin North Am. 2012; 43(5):e23-34.

11. Leslie IJ et al. High cup angle and micro separation increase the wear of hip surface replacements. Clin OrthopRelat Res. 2009; 467(9):2259-65

12. Yamaguchi $\mathrm{M}$ et al. The spatial location of impingement in total hip arthroplasty. J Arthroplasty. 2000; 15(3):305-313.

13. Shon WY et al. Impingement in Total Hip Arthroplasty: A Study of Retrieved Acetabular Components. J Arthroplasty. 2005; 20(4):427-435.

14. Devitt A, O'Sullivan T, Quinlan W. 16- to 25-year followup study of cemented arthroplasty of the hip in patients aged 50 years or younger. J Arthroplasty. 1997; 12:479-89.

15. Kawalkar AC, Badole CM, Phadke A. Midterm results of cementless total hip arthroplasty in young. Journal of Orthopaedics and Allied Sciences. 2016; 4(1):30. 